\title{
Multi-Indices Quantification for Left Ventricle via DenseNet and GRU-Based Encoder-Decoder with Attention
}

\author{
Zhi Liu $\mathbb{D}^{1}{ }^{1}$ Yunhua Lu, ${ }^{1}$ Xiaochuan Zhang, ${ }^{1}$ Sen Wang, ${ }^{1}$ Shuo Li, ${ }^{2,3}$ and Bo Chen ${ }^{4}$ \\ ${ }^{1}$ School of Artificial Intelligence, Chongqing University of Technology, Chongqing 400054, China \\ ${ }^{2}$ Department of Medical Imaging, Western University, London, ON, Canada N6A3K7 \\ ${ }^{3}$ The Digital Imaging Group of London, London, ON, Canada N6A $3 K 7$ \\ ${ }^{4}$ School of Health Science, Western University, London, ON, Canada N6A3K7 \\ Correspondence should be addressed to Zhi Liu; liuzhi@cqut.edu.cn
}

Received 25 June 2020; Revised 11 August 2020; Accepted 10 February 2021; Published 20 February 2021

Academic Editor: Zhile Yang

Copyright $\odot 2021$ Zhi Liu et al. This is an open access article distributed under the Creative Commons Attribution License, which permits unrestricted use, distribution, and reproduction in any medium, provided the original work is properly cited.

\begin{abstract}
More and more research on left ventricle quantification skips segmentation due to its requirement of large amounts of pixel-bypixel labels. In this study, a framework is developed to directly quantify left ventricle multiple indices without the process of segmentation. At first, DenseNet is utilized to extract spatial features for each cardiac frame. Then, in order to take advantage of the time sequence information, the temporal feature for consecutive frames is encoded using gated recurrent unit (GRU). After that, the attention mechanism is integrated into the decoder to effectively establish the mappings between the input sequence and corresponding output sequence. Simultaneously, a regression layer with the same decoder output is used to predict multi-indices of the left ventricle. Different weights are set for different types of indices based on experience, and 12-norm is used to avoid model overfitting. Compared with the state-of-the-art (SOTA), our method can not only produce more competitive results but also be more flexible. This is because the prediction results in our study can be obtained for each frame online while the SOTA only can output results after all frames are analyzed.
\end{abstract}

\section{Introduction}

Great importance has been attached to the research of left ventricle quantification during the identification and diagnosis of cardiac disease in clinical routine. It can evaluate the current situation of patients and then make appropriate and correct judgments on the prognosis and outcome of the disease. In recent years, Cardiac Magnetic Resonance (CMR) has become a crucial imaging modality in clinical cardiology practice due to its high signal-to-noise ratio, noninvasive imaging to cardiac chambers, no need for geometric assumptions, and great vessels $[1,2]$. Although much effort has been devoted to left ventricle quantification over the last several decades [2-8], it remains in the research stage and the reported algorithms are still not robust and flexible enough to support clinic practice due to the complexity of medical imaging. Therefore, left ventricle quantification is still acknowledged as a challenge with much room for improvements in robustness, flexibility, and accuracy.

In general, left ventricle quantification can be implemented according to Simpson's rule [9] after epicardial and endocardial contours are delineated on the short-axis slices $[5,8]$. However, due to the characteristics of cardiac MR images and the great variability of the images among patients, left ventricle segmentation in MRI still remains a challenge. On the one hand, segmentation complexity depends on the slice level of the image. For example, it is more difficult for Apical and basal slice images to segment than that of midventricular. Furthermore, segmentation-based methods usually introduce two phases to quantify the left ventricle, resulting in difficulty to optimize the framework as a whole. At present, the end-to-end framework is increasingly popular with the development of deep learning technology $[3,10]$. 
In this paper, in order to achieve a reliable and accurate solution to quantify left ventricle from short-axis view cardiac MR images, an elegant framework based on DenseNet [11] and GRU- [12] based encoder-decoder with attention is built. Specifically, the structural parameters such as myocardium and cavity areas, region wall thicknesses and cavity dimensions (Figure 1) will be evaluated for each frame in our approach. The main contributions of this work are as follows.

Firstly, a shared and end-to-end framework was proposed to directly quantify left ventricle indices without segmentation. All frames share the DenseNet to extract their spatial feature, making the model more concise and avoid overfitting.

Secondly, in order to catch the temporal information across consecutive frames, the extracted features of consecutive frames are encoded by GRU blocks. This is because GRU has a great ability to retain long-term dependencies in sequential data, and, in comparison with its sibling LSTM, it has similar accuracy with fewer parameters and faster speed.

Finally, the encoded temporal features of consecutive frames and each frame were mapped to final representations via a decoder constructed by GRU blocks with attention, which imparts the decoder to focus on local or global features. The final representations are input to the regression layer to quantify indices of the frame.

The rest of this paper is organized as follows: In Section 2 , some related works are discussed. Then, deep encoderdecoder recurrent neural network Models with attention is proposed in Section 3. Experimental settings and details about the dataset are introduced in Section 4. Results and detailed discussions are shown in Section 5. Section 6 is the conclusion.

\section{Related Works}

The final goal of left ventricle research is to obtain structural and functional parameters for accurate identification and diagnosis of cardiac diseases. These parameters may be a low level of myocardium and cavity areas, region wall thicknesses, and cavity dimensions, or a high level of End Systole Volume (ESV), End Diastole Volume (EDV), and Eject Fraction (EF). Plenty of left ventricle quantification methods have been reported in the past few decades, which mainly can be divided into 2 categories: segmentation-based methods and regressionbased methods.

2.1. Segmentation-Based Methods. In practical clinical medicine, the doctors generally obtain reliable quantification by manually contouring the borders of myocardium, which is time-consuming and unfavourable for diagnostic automation [2]. In addition, manual contouring is prone to intraand interobserver variability. Therefore, much research about automated segmentation methods has been carried out, much of which focuses on the segmentation on left ventricle based on mathematical calculations or deep learning method. This is because every frame of all slices can be quantized according to Simpson's Rule after segmentation.

2.1.1. Segmentation Based on Mathematical Calculations. Segmentation based on mathematical calculations delineates the endo- and/or epicardial boundaries in all frames of a cardiac sequence according to the shape characteristic of left ventricle. However, in order to increase the robustness and accuracy, most of the mathematical segmentation requires a prior knowledge, problematic assumptions, or user interaction [13-15].

2.1.2. Deep Learning-Based Segmentation. A lot of research has been dedicated to the deep learning-based segmentation since the AlexNet model obtained remarkable classification accuracy in the ImageNet Large Scale Visual Recognition Challenge in 2012 [16]. Deep learning-based Segmentation for left ventricle keeps up with the development of deep learning technology in segmentation. The representative methods consist of CNN $[4,5,17]$, sliding window [18-20], Full Connected Network [6, 21-23], U-Net [7, 8, 24], and 3D Convolution [25-27].

As long as the segmentation of all frames in a cardiac sequence is accomplished, the structural and functional parameters can be calculated according to MRI parameters and fixed methods such as Simpson's Rule. However, numerous mathematics-based segmentation requires prior knowledge or user intervention, while deep learning-based segmentation requires a large number of training samples. Each pixel of these samples needs to be labeled to obtain robustness and good results.

2.2. Regression-Based Methods. In order to reduce large tedious labeling work and user intervention, much research skips the segmentation process and then directly obtains the cardiac parameters by regression based on the extracted features from cardiac MRIs. Two kinds of regression methods can be classified based on hand-crafted features and automatic deep learning features separately. In earlier years, almost all direct regression methods extract hand-crafted features for regression of cardiac parameters due to the limitation of computing power. These methods usually follow a common two-phase framework: cardiac image representation and indices prediction. First, cardiac images are usually represented by hand-crafted features or features obtained by unsupervised learning, such as Bhattacharyya coefficients between image distributions $[28,29]$, appearance features [30], multiple low-level image features [31], as well as features from multiscale convolution deep belief network [32], and supervised descriptor learning [33]. Then, cardiac indices are estimated from these features with a separate regression model.

Since 2012, with the development of deep learning technology, the regression based on automatic deep learning features has sparked an impressive research effort, with promising performances and a breadth of techniques $[4,6,10,30,32,33]$. With its unique capabilities of end-to- 
end, pipeline simplicity, and no loss of information, regression based on automatic deep learning is increasingly favored by researchers. In this paper, we follow this trend and develop an end-to-end framework to quantify left ventricle indices directly without segmentation.

\section{Methods}

The proposed model consists of three tightly integrated modules. Firstly, the deep convolution neural network DenseNet is used for cardiac image (frame) representation, and then an encoder-decoder structure built using GRU blocks is deployed for temporal dynamic modeling of cardiac sequences. To emphasize the contribution of the current input frame, an attention model is added to the decoder network. The outputs of encoder-decoder module are fed into a multitask learning module for left ventricle indices estimation.

3.1. Framework and Problem Formulation. The overview of the proposed framework is shown in Figure 2. To better describe the problem, we use $X=x_{s}^{f}$ to represent the entire dataset and the left ventricle indices are denoted as $\operatorname{area}_{s}^{f}, d i m_{s}^{f}, r w t_{s}^{f}$, where $s=1,2, \ldots, n_{s}$ indicates the subject and $f=1,2, \ldots, n_{f}$ indicates the frame. While $\operatorname{area}_{s}^{f}$ is a two-dimensional vector which describes the area of myocardium and left ventricle cavity, $d i m_{s}^{f}$ is a threedimensional vector which describes three left ventricle cavity dimensions, and $r w t_{s}^{f}$ is a six-dimensional vector which describes the regional wall thickness in six directions. Then, the proposed framework $\mathrm{f}_{f}$ can be modelled as a regression problem with input $\left\{x_{s}^{f_{i-n_{l}+1}}, x_{s}^{f_{i-n_{l}+2}}, \ldots, x_{s}^{f_{i}}\right\}$ and output area $f_{s}^{f_{i}}, d i m_{s}^{f_{i}}, r w t_{s}^{f_{i}}$. That is, $n_{l}$ consecutive frames $\left(x_{s}{ }^{i-n_{l}+1}, \ldots, x_{s}^{f_{i}}\right)$ before the current frame are used to predict the indices of the current frame $\left(x_{s}^{f_{i}}\right)$. For the input $\left\{x_{s}^{f_{i-n_{l}+1}}, \ldots, x_{s}^{f_{i}}\right\}, n_{f}$ frames are regarded as a circular sequence since heartbeat is a cycle process. According to this perspective, $x_{s}^{f_{i-\eta_{l}+1}}$ will be substituted by $x_{s}{ }^{i-n_{l}+1+n_{f}}$ if $i-n_{l}+1<=0$, in which $n_{f}$ equals 20 on DIG-Cardiac database [4]. The algorithm description is presented in Algorithm 1 in order to state the framework more clearly.

In the proposed framework, both the encoder and decoder apply a two-layer GRU to the corresponding input sequence. For each element in the input sequence, each layer computes the following function:

$$
\begin{aligned}
& r_{t}=\sigma\left(W_{i r} x_{t}+b_{i r}+W_{h r} h_{(t-1)}+b_{h r}\right), \\
& z_{t}=\sigma\left(W_{i z} x_{t}+b_{i z}+W_{h z} h_{(t-1)}+b_{h z}\right), \\
& n_{t}=\tanh \left(W_{i n} x_{t}+b_{i n}+r_{t}^{*}\left(W_{h n} h_{(t-1)}+b_{h n}\right)\right), \\
& h_{t}=\left(1-z_{t}\right) * n_{t}+z_{t}^{*} h_{(t-1)} .
\end{aligned}
$$

where $h_{t}$ is the hidden state at time $t, x_{t}$ is the input at time $t$, $h_{(t-1)}$ is the hidden state of the layer at time t-1 or the initial hidden state at time 0 , and $r_{t}, z_{t}, n_{t}$ are the reset, update, and new gates, respectively. $\sigma$ is the sigmoid function, and ${ }^{*}$ is the Hadamard product.

3.2. Spatial Feature Extraction Using DenseNet. DenseNet, with compelling advantages such as alleviating the vanishing-gradient problem, strengthening feature propagation, encouraging feature reuse, and substantially reducing the number of parameters, has the excellent capability of capturing spatial features. Compared with ResNet, the $l$-th layer receives the feature maps of all preceding layers $x_{0}, x_{1}, \ldots, x_{l-1}$, as input to further improve the information flow between layers:

$$
x_{l}=H_{l}\left(\left[x_{0}, x_{1}, \ldots, x_{l-1}\right]\right),
$$

where $\left[x_{0}, x_{1}, \ldots, x_{l-1}\right]$ refers to the concatenation of the feature maps produced in layers $0, \ldots, l-1$. This encourages feature reuse throughout the network and leads to more compact models and consistently the most parameter efficient variant. In this study, DenseNet is used to extract spatial features for each frame. Our DenseNet in Figure 2 contains a convolutional layer (conv1) capturing low-level features from images, three DenseBlocks (one DenseBlock includes one Bottleneck (0) and one Bottleneck (1)), and two Transition layers between adjacent dense blocks. Table 1 lists more details of the DenseNet network.

To better interpret what the DenseNet learns about from the frames, the feature map (Figure 3) of DenseNet in different layers is visualized. And the activation feature map of the second convolution layer in the first BottleNeck for each DenseBlock is presented. From the visualization, it can be seen that the shallow layer can preserve detailed information such as contour and texture. While the layers go deeper, they will extract more abstract features so that we can understand their feature map with the naked eye. To state conveniently, the convolution layer from the first, second, and third DenseBlock are named DB1Conv, DB2Conv, and DB3Conv. The filters from DB1Conv capture low-level visual features from cardiac frames, such as the low-frequency appearance of the ventricles and textures of the ventricles. Taking the low-level features of DB1Conv as input, subsequent blocks (DB2Conv) extract more complex cardiac information by combining low-level features. Gradually, the high-frequency noise or textures are discarded in this procedure. Lastly, the detailed spatial information of the feature maps in DB3Conv becomes increasingly blurred. Instead, they extract more abstract and higher level features in each local area from the preceding feature maps during the training procedure.

3.3. Temporal Information Encoding and Decoding Using GRUs with Attention. Heartbeat is a continuous cycle process from systole to diastole. The previous frame sequence is beneficial to parameters' evaluation of the current frame. For sequence modelling, compared with LSTM, GRU is easier to modify and does not need memory units and thus results in less training time with the same performance as that of LSTM. In this paper, GRUs are used in modelling the input sequence of several continuous frames. Then, the modelled sequence will be decoded to predict the parameters of current frames. Human beings can quickly understand the parameters trend and relationships between the input sequence and the output with the naked eye. However, the neural network is not so direct to detect these relationships 


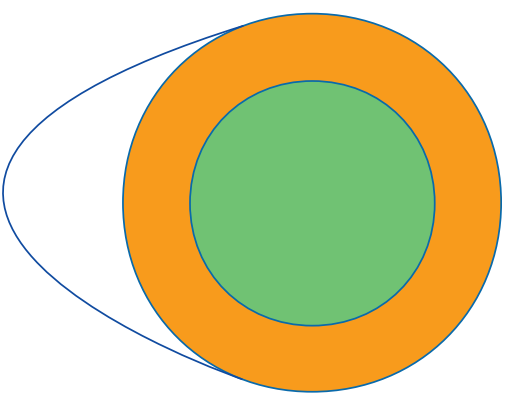

(a)

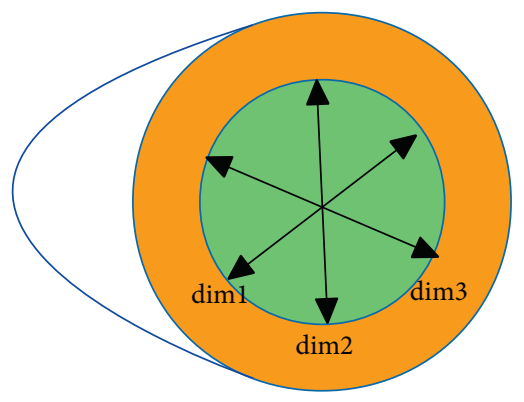

(b)

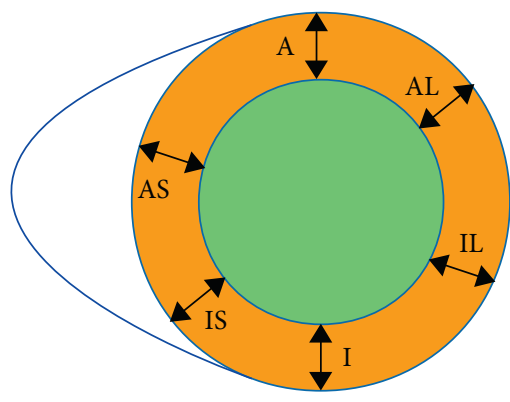

(c)

Figure 1: The schematic diagram of evaluated indices [4]. (a) Cavity area and myocardium area in $\mathrm{mm}^{2}$. (b) Dimensions of the cavity of three directions (ISAL, IA, and ILAS) in $\mathrm{mm}$. (c) Regional wall thickness in $\mathrm{mm}$, staring from the anteroseptal segment in a counterclockwise direction (IS, I, IL, AL, A, and AS).

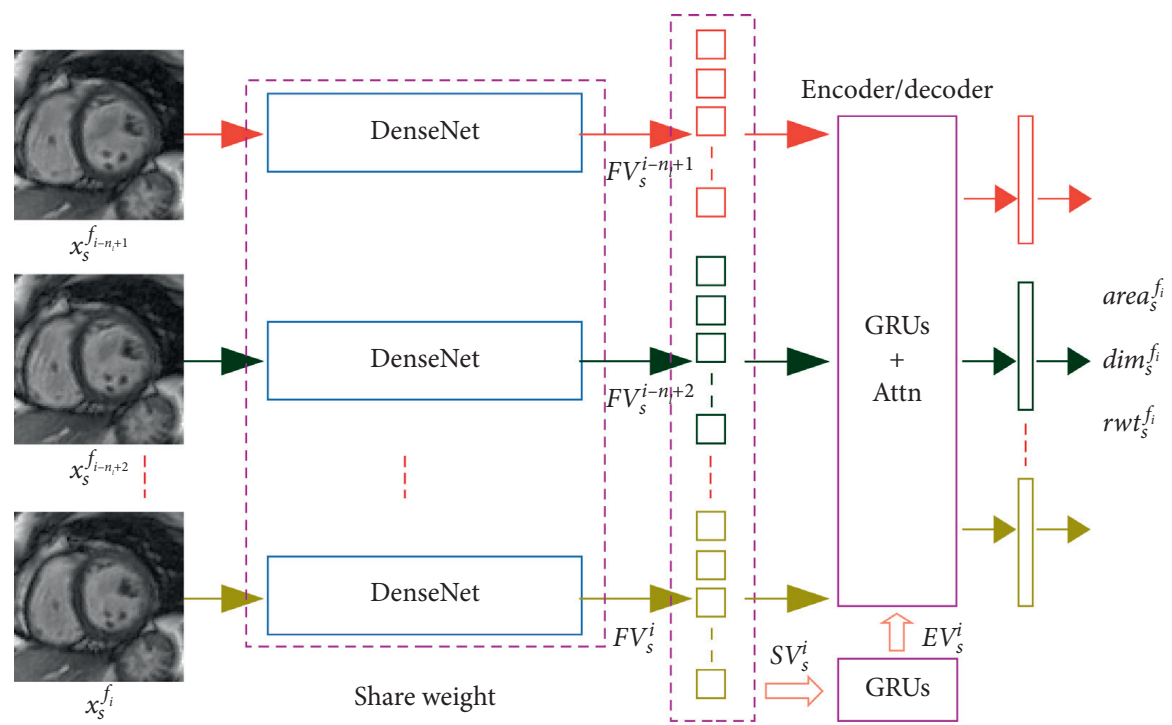

FIGURE 2: The proposed framework for left ventricle quantification. The GRUs constitute the encoder and the GRUs + Attn constitute the decoder.

Model: DenseNet, Encoder, and Decoder as shown in Figure 2

Input: $n_{l}$ consecutive frames $x_{s}^{f_{i-n_{l}+1}}, x_{s}^{f_{i-n_{l}+2}}, \ldots, x_{s}^{f_{i}}$

Output: $\operatorname{area}_{s}^{f_{i}}, \operatorname{dim}_{s}^{f_{i}}, r w t_{s}^{f_{i}}$ at frame $f_{i}$

Algorithms: Predict the indices area $a_{s}^{f_{i}}, \operatorname{dim}_{s}^{f_{i}}, r w t_{s}^{f_{i}}$ for frame $f_{i}$

(1) $S V_{s}^{i}=/ /$ Sequence Vector

(2) for $t=i-n_{l}+1$ to $i$ do

(3) $F V_{s}^{t}=\operatorname{DenseNet}\left(x_{s}^{f_{t}}\right) / /$ Feature Vector

(4) $S V_{s}^{i}=S V_{s}^{i} \& F V_{s}^{t} / / \&$ is vector concatenation

(5) end for

(6) $E V_{s}^{i}=\operatorname{Encoder}\left(S V_{s}^{i}\right) / /$ Encoded Vector

(7) $\left[\operatorname{area}_{s}^{f_{i}}, \operatorname{dim}_{s}^{f_{i}}, r w t_{s}^{f_{i}}\right]=$

(8) for $t=i-n_{l}+1$ to $i$ do

(9) $\left[\right.$ area $\left.a_{s}^{f_{i}}, \operatorname{dim}_{s}^{f_{i}}, r w t_{s}^{f_{i}}\right]=$ Decode $r\left(F V_{s}^{t}, E V_{s}^{i}\right)$

(10) end for

(11) return $\left[\operatorname{area}_{s}^{f_{i}}, \operatorname{dim}_{s}^{f_{i}}, r w t_{s}^{f_{i}}\right] / /$ The last output of Decoder

Algorithm 1: Left ventricle indices quantification for a frame. 
automatically. Thus, the attention mechanism is integrated into the decoder to learn these relationships through gradient descent and backpropagation. Figure 4 presents the decoding process with the attention mechanism.

\section{Experiment and Configuration}

4.1. Dataset. Our approach is evaluated on the DIG-Cardiac database [4]. It consists of 145 cardiac MR images collected from 3 hospitals affiliated with two health care centers (London Healthcare Center and St. Josephs HealthCare). The subjects' ages were from 16 yrs to $97 \mathrm{yrs}$, with an average of $58.9 \mathrm{yrs}$. The pixel spacings of the MR images range from $0.6836 \mathrm{~mm} /$ pixel to $2.0833 \mathrm{~mm} / \mathrm{pixel}$, with a mode of $1.5625 \mathrm{~mm} /$ pixel. Diverse pathologies are in presence including regional wall motion abnormalities, myocardial hypertrophy, mildly enlarged left ventricle, atrial septal defect, and left ventricle dysfunction. Each subject contains $n_{f}=20$ frames throughout a cardiac cycle. In each frame, the left ventricle is divided into equal thirds (basal, midcavity, and apical) perpendicular to the long axis of the heart following the standard AHA prescription and a representative midcavity slice is selected for this database. All cardiac images undergo several preprocessing steps, including landmark labeling, rotation, ROI cropping, and resizing. The resulted images are approximately aligned with a dimension of $80 \times 80$. Then, these cardiac images are manually contoured to obtain the epicardial and endocardial borders, which are double-checked by two experienced cardiac radiologists (A. Islam and M. Bhaduri). The ground truth values of left ventricle indices and cardiac phase can be easily obtained from the two borders. The values of RWT and cavity dimensions are normalized by the image dimension, while the areas are normalized by the pixel number (6400). During evaluation, the obtained results need to be converted into physical thickness (in $\mathrm{mm}$ ) and area (in $\mathrm{mm}^{2}$ ) by reversing the resizing procedure and multiplying the pixel spacing for each subject.

4.2. Implementation and Configuration. Our model is built, trained, and tested by Pytorch [34]. The neural network is trained by minimizing L1_Loss as the objective function. Stochastic gradient descent is used to optimize the object function. The model is initialized with the default methods in Pytorch. That is, convolution layers and linear layers are initialized using Xavier methods and with random values sampled from a uniform distribution, respectively. The initial learning rate is $1 \times 10^{-3}$ which was gradually reduced to $1 \times 10^{-5}$ during training. The momentum is 0.9 and the weight decay is $1 \times 10^{-4}$. In our experiments, 5 -fold crossvalidation is employed for performance evaluation and comparison. The dataset is divided into 5 groups according to subject number from 1 to 145 , each containing 29 subjects. In each experiment, four groups are employed to train the prediction model, and the remaining group is used for the test. This procedure is repeated five times until the indices of all subjects are obtained. The model is trained on a server with the following settings: CPU-Intel(R) Core(TM)
i7-9700K CPU @ 3.60 GHz (8 CPUs), Memory-16384 MB RAM, GPU-NVIDIA GeForce RTX 2080 Ti with $11048 \mathrm{MB}$ Dedicated Memory. Given the batch size of 8 and the number of input frames of 3 , it takes more than 20 hours to complete the training run of 200 epochs.

\subsection{Evaluation Criteria and Data Conversion}

4.3.1. Evaluation Criteria. The proposed framework is evaluated in terms of mean absolute error (MAE) and coefficient between the ground truth and prediction. That is, for all frames in the cardiac cycle, MAE and coefficient are computed for each left ventricle index. The MAE and coefficient are computed according to equations (2) and (3). The final MAE and coefficient are averaged by all frames of testing subjects:

$$
\begin{aligned}
\text { MAE } & =\frac{1}{N} \sum_{i=1}^{N}\left(y_{i}-\widehat{y}_{i}\right), \\
\rho & =\frac{2 \sum_{i=1}^{N}\left(y_{i}-m\right)\left(\hat{y}_{i}-\widehat{m}\right)}{\sum_{i=1}^{N}\left(\left(y_{i}-m\right)^{2}+\left(\hat{y}_{i}-\widehat{m}\right)^{2}\right)},
\end{aligned}
$$

where $m=(1 / N) \sum_{i=1}^{N} y_{i}$ and $\widehat{m}=(1 / N) \sum_{i=1}^{N} \hat{y}_{i}$.

4.3.2. Data Conversion. The provided values of Rwts, Dims, and Areas in the DIG-Cardiac database are normalized values according to actual measurements. This Original Value $(\mathrm{OV})$ is utilized to train and test the model. After that, the output values are converted to Physical Measurements (PM) to evaluate the proposed method according to the database specification. For Areas, the formula is as follows:

$$
\mathrm{PM}=6400 \times \mathrm{OV} \times(\text { ratio_resize_inverse } \times \text { pix_spacing })^{2} \text {. }
$$

For Dims and Rwts, the formula is as follows:

$$
\mathrm{PM}=80 \times \mathrm{OV} \times(\text { ratio_resize_inverse } \times \text { pix_spacing }),
$$

where pix_spacing is the original pixel spacing (in $\mathrm{mm}$ ) of MR images and ratio_resize_inverse is the ratio to reverse the resize procedure provided with DIG-Cardiac database. 6400 and 800 are normalizing parameters used in the database.

\section{Results and Analysis}

5.1. Performance Evaluation of the Proposed Method. After 5-fold cross-validation experiments, the prediction results of multiple indices are shown in Table 2 . In the experiments, the parameter SeqLen is set to 3 which is small for computation efficiency. The learned model can predict all the indices well for each frame. The averages MAE for Area, Dim, and Rwt are $209.74 \mathrm{~mm}^{2}, 2.48 \mathrm{~mm}$, and $1.57 \mathrm{~mm}$. Significantly, the maximums of these indices for Area, Dim, and $R w t$ in the DIG-Cardiac database reach $4936 \mathrm{~mm}^{2}$, $81.0 \mathrm{~mm}$, and $24.4 \mathrm{~mm}$, respectively. Good performance is 
TABLE 1: The network parameters for spatial feature extraction.

\begin{tabular}{|c|c|c|c|c|c|c|c|}
\hline Module & Pytorch Layer & In channels & Out channels & Kenel size & Stride & Padding & Bias \\
\hline conv1 & Conv2d & 1 & 16 & $(3,3)$ & $(1,1)$ & $(1,1)$ & False \\
\hline \multirow{4}{*}{ Bottleneck (0) } & BatchNorm2d & 16 & - & - & - & - & - \\
\hline & Conv2d & 16 & 32 & $(1,1)$ & $(1,1)$ & $(0,0)$ & False \\
\hline & BatchNorm $2 \mathrm{~d}$ & 32 & - & - & - & - & - \\
\hline & Conv2d & 32 & 8 & $(3,3)$ & $(1,1)$ & $(1,1)$ & False \\
\hline \multirow{4}{*}{ Bottleneck (1) } & BatchNorm2d & 24 & - & - & - & - & - \\
\hline & Conv2d & 24 & 32 & $(1,1)$ & $(1,1)$ & $(0,0)$ & False \\
\hline & BatchNorm $2 \mathrm{~d}$ & 32 & - & - & - & - & - \\
\hline & Conv2d & 32 & 8 & $(3,3)$ & $(1,1)$ & $(1,1)$ & False \\
\hline \multirow{2}{*}{ Transition } & BatchNorm2d & 32 & - & - & - & - & - \\
\hline & Conv2d & 32 & 16 & $(1,1)$ & $(1,1)$ & $(0,0)$ & False \\
\hline bn1 & BatchNorm2d & 32 & - & - & - & - & - \\
\hline Pool & avg_pool2d & 32 & - & $(4,4)$ & $(4,4)$ & $(0,0)$ & - \\
\hline $\mathrm{Fc}$ & Linear & 512 & 128 & - & - & - & - \\
\hline
\end{tabular}
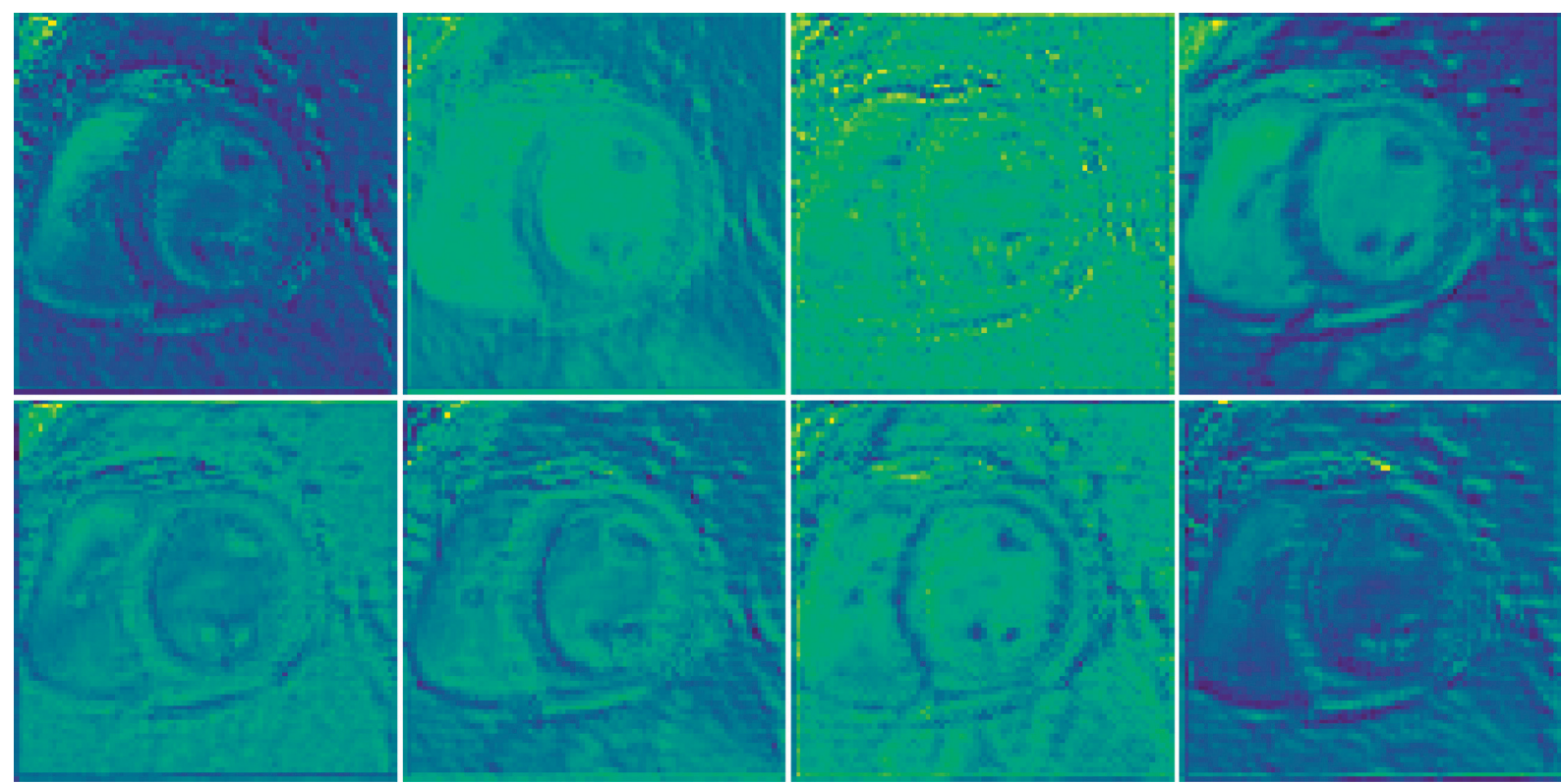

(a)

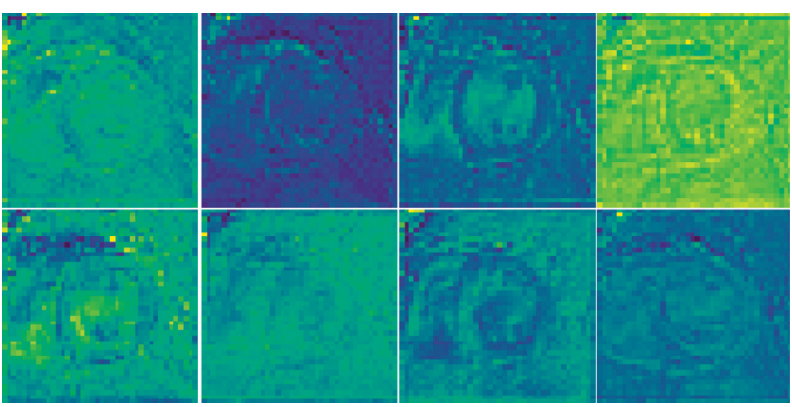

(b)

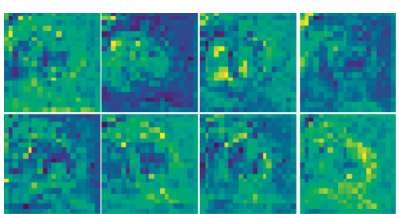

(c)

FIGURE 3: Feature map visualization for DenseNet. Feature maps from the first, second, and third DenseBlock are presented in (a) $8 \times 80 \times 80$, (b) $8 \times 20 \times 20$, and (c) $8 \times 40 \times 40$ in which the number of filters is 8 and the receptive field becomes smaller with the layers going deeper. 


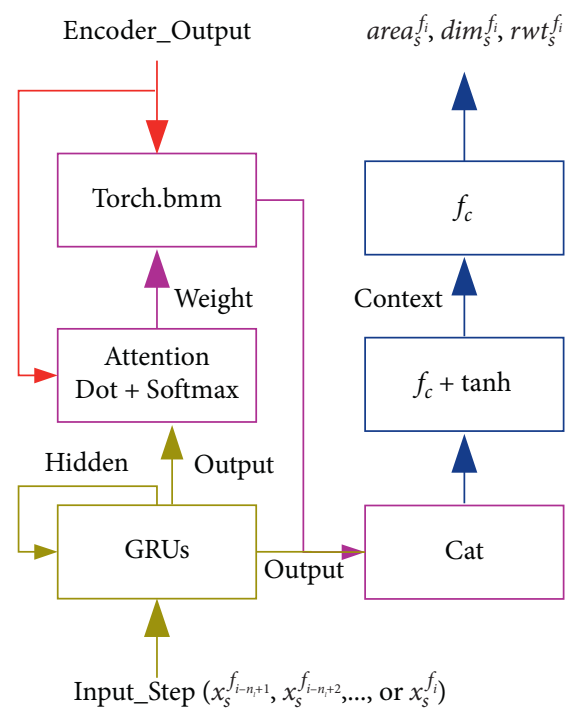

FIGURE 4: Decoder built in GRUs with attention. Encoder_Output is the output from the encoder which learns temporal features from the input cardiac frame sequences and Input_Step is one of the frames in the input cardiac frame sequence.

TABle 2: Performance evaluation of the proposed method.

\begin{tabular}{|c|c|c|c|c|}
\hline Indices & MAE & Coef. & MAE AVG & Coef. AVG \\
\hline $\begin{array}{l}\text { Area cavity } \\
\text { Area myocardium }\end{array}$ & $\begin{array}{l}179.72 \\
239.76\end{array}$ & $\begin{array}{l}0.8893 \\
0.8526\end{array}$ & 209.74 & 0.8709 \\
\hline $\begin{array}{l}\text { Dim } 1 \\
\text { Dim } 2 \\
\text { Dim } 3\end{array}$ & $\begin{array}{l}2.38 \\
2.23 \\
2.83\end{array}$ & $\begin{array}{l}0.8678 \\
0.8765 \\
0.8351\end{array}$ & 2.48 & 0.8598 \\
\hline $\begin{array}{l}\text { Rwt-IS } \\
\text { Rwt-I } \\
\text { Rwt-IL } \\
\text { Rwt-AL } \\
\text { Rwt-A } \\
\text { Rwt-AS }\end{array}$ & $\begin{array}{l}1.65 \\
1.65 \\
1.82 \\
1.52 \\
1.15 \\
1.63 \\
\end{array}$ & $\begin{array}{l}0.7753 \\
0.6504 \\
0.6563 \\
0.7403 \\
0.7702 \\
0.7479 \\
\end{array}$ & 1.57 & 0.7234 \\
\hline
\end{tabular}

attributed to the GRU, which has the ability to extract features and preserve more temporal feature or variation trend. And both the temporal feature from frame sequences and spatial feature from the current frame are conducive to the prediction improvement.

5.2. Comparison with SOTA and Other Baselines. Our framework provides competitive results compared to SOTA (see details in Table 3). The MAE of Dim is slightly lower than that of SOTA. In addition, our approach shows more flexibility than SOTA because the former can predict the indices frame by frame and support the input frames of any number, while the latter only can predict the indices of all frames at once by inputting the whole frame sequence, which results in the adjustment of the framework when MRI slice has a different number of frames. To verify the effectiveness of temporal modelling and attention mechanism, a baseline work is developed and compared with the proposed framework. In the baseline, temporal modeling is discarded completely and only DenstNet is used to extract spatial
TABle 3: Performance evaluation with SOTA and baselines.

\begin{tabular}{lcccccc}
\hline \multirow{2}{*}{ Model } & \multicolumn{2}{c}{ Area } & \multicolumn{2}{c}{ Dim } & \multicolumn{2}{c}{ Rwt } \\
& MAE & Coef. & MAE & Coef. & MAE & Coef. \\
\hline SpaNet & 285.85 & 0.7463 & 3.21 & 0.7748 & 2.22 & 0.5490 \\
[4] & 180 & 0.945 & 2.51 & 0.925 & 1.39 & 0.768 \\
Proposed & 209.74 & 0.8709 & $\mathbf{2 . 4 8}$ & 0.8598 & 1.57 & 0.7234 \\
\hline
\end{tabular}

features for each frame. The extracted features are used to predict left ventricle indices for each frame. For ease of following description, the baseline is denoted as SpaNet, meaning that only spatial features are extracted for each frame. Table 3 presents the detailed experimental results for the baseline model, SOTA, and proposed framework. From the table, it can be seen that the proposed method outperforms the baseline model greatly. The reason may be that temporal modelling provides more information which is complementary to spatial features and the attention mechanism makes the model focus on the most discriminate part of the input. 


\section{Conclusion}

In our study, a framework is developed for quantifying left ventricle indices. And the framework not only preserves the spatial information for each frame using DenseNet but also keeps the temporal information by encoding extracted features for consecutive frames using GRU. More importantly, to effectively map the input to the output, a decoder based on GRU with attention is designed. The final representation is obtained by inputting encoded temporal features of consecutive frames and each frame in it. Further, competitive results can be obtained on the DIG-Cardiac database using 5-fold cross-validation which is the same as SOTA. But our approach predicts the left ventricle indices frame-byframe while the SOTA predicts the indices after all frames are analyzed. Therefore, our method is more flexible because it supports any number of frame inputs. We insist that longer frame sequences are more favorable for left ventricle quantification. More experiments will be done in future work to testify the influence of different length frame sequences on the performance of indices prediction for left ventricle.

\section{Data Availability}

The DIG-Cardiac database used to support the findings of this study is available from the corresponding author upon request.

\section{Conflicts of Interest}

The authors declare that they have no conflicts of interest.

\section{Acknowledgments}

This work was supported by the Natural Science Foundation of Chongqing, China (Grant nos. cstc2019jcyj-msxmX0487 and cstc2019jcyj-msxmX0544) and the National Science Foundation of China (Grant no. 61702063). The authors also acknowledge the support of China Scholarship Council (Grant no. 201808505123).

\section{References}

[1] P. Peng, K. Lekadir, A. Gooya, L. Shao, S. E. Petersen, and A. F. Frangi, "A review of heart chamber segmentation for structural and functional analysis using cardiac magnetic resonance imaging," Magnetic Resonance Materials in Physics, Biology and Medicine, vol. 29, no. 2, pp. 155-195, 2016.

[2] A. Suinesiaputra, D. A. Bluemke, B. R. Cowan et al., "Quantification of LV function and mass by cardiovascular magnetic resonance: multi-center variability and consensus contours," Journal of Cardiovascular Magnetic Resonance, vol. 17, no. 1, p. 63, 2015.

[3] J. J. Mantilla, J. L. Paredes, J.-J. Bellanger, E. Donal, C. Leclercq, and M. Garreau, "Discriminative dictionary learning for local LV wall motion classification in cardiac MRI," Expert Systems with Applications, vol. 129, pp. 286-295, 2019.
[4] W. Xue, G. Brahm, S. Pandey, S. Leung, and S. Li, "Full left ventricle quantification via deep multitask relationships learning," Medical Image Analysis, vol. 43, pp. 54-65, 2018.

[5] X. Du, R. Tang, S. Yin, Y. Zhang, and S. Li, "Direct segmentation-based full quantification for left ventricle via deep multi-task regression learning network," IEEE Journal of Biomedical and Health Informatics, vol. 23, no. 3, pp. 942-948, 2018.

[6] F. Liao, X. Chen, X. Hu, and S. Song, "Estimation of the volume of the left ventricle from MRI images using deep neural networks," IEEE Transactions on Cybernetics, vol. 49, no. 2, pp. 495-504, 2017.

[7] Q. Tao, W. Yan, Y. Wang et al., "Deep learning based method for fully automatic quantification of left ventricle function from cine MR images: a multivendor, multicenter study," Radiology, vol. 290, no. 1, pp. 81-88, 2018.

[8] E. Abdelmaguid, J. Huang, S. Kenchareddy et al., "Left ventricle segmentation and volume estimation on cardiac MRI using deep learning," 2018, https://arxiv.org/ftp/arxiv/ papers/1809/1809.06247.pdf.

[9] L. K. Wandelt, J. T. Kowallick, A. Schuster et al., "Quantification of left atrial volume and phasic function using cardiovascular magnetic resonance imaging-comparison of biplane area-length method and Simpson's method," The International Journal of Cardiovascular Imaging, vol. 33, no. 11, pp. 1761-1769, 2017.

[10] J. Degrave, "Using deep learning to estimate systolic and diastolic volumes from MRI-images," 2016, https://www. kuleuven-kulak.be/benelearn/papers/Benelearn_2016_paper_ 19.pdf.

[11] G. Huang, Z. Liu, L. Van Der Maaten, and K. Q. Weinberger, "Densely connected convolutional networks," in Proceedings of the IEEE conference on computer vision and pattern recognition, pp. 4700-4708, Honolulu, HI, USA, July 2017.

[12] J. Chung, C. Gulcehre, K. Cho, and Y. Bengio, "Empirical evaluation of gated recurrent neural networks on sequence modeling," 2014, https://arxiv.org/abs/1412.3555.

[13] A. Gupta, L. Von Kurowski, A. Singh et al., "Cardiac MR image segmentation using deformable models," in Proceedings of the Computers in Cardiology Conference, pp. 747-750, London, UK, September 1993.

[14] Y. Wu, Y. Wang, and Y. Jia, "Segmentation of the left ventricle in cardiac cine MRI using a shape-constrained snake model," Computer Vision and Image Understanding, vol. 117, no. 9, pp. 990-1003, 2013.

[15] I. B. Ayed, H.-M. Chen, K. Punithakumar, I. Ross, and S. Li, "Max-flow segmentation of the left ventricle by recovering subject-specific distributions via a bound of the Bhattacharyya measure," Medical Image Analysis, vol. 16, no. 1, pp. 87-100, 2012.

[16] A. Krizhevsky, I. Sutskever, and G. E. Hinton, "Imagenet classification with deep convolutional neural networks," in Proceedings of the Advances in Neural Information Processing Systems, pp. 1097-1105, Lake Tahoe, Nevada, USA, December 2012.

[17] C. Xu, L. Xu, Z. Gao et al., "Direct delineation of myocardial infarction without contrast agents using a joint motion feature learning architecture," Medical Image Analysis, vol. 50, pp. 82-94, 2018.

[18] V. Jain, J. F. Murray, F. Roth et al., "Supervised learning of image restoration with convolutional networks," in Proceedings of the 2007 IEEE 11th International Conference on Computer Vision, pp. 1-8, Rio De Janeiro, Brazil, October 2007. 
[19] F. Yu and V. Koltun, "Multi-scale context aggregation by dilated convolutions," 2015, https://arxiv.org/abs/1511.07122.

[20] K. Lee, A. Zlateski, V. Ashwin, and H. S. Seung, "Recursive training of 2D-3D convolutional networks for neuronal boundary prediction," in Proceedings of the Advances in Neural Information Processing Systems, pp. 3573-3581, Montreal, Canada, December 2015.

[21] M. Khened, V. A. Kollerathu, and G. Krishnamurthi, "Fully convolutional multi-scale residual densenets for cardiac segmentation and automated cardiac diagnosis using ensemble of classifiers," Medical Image Analysis, vol. 51, pp. 21-45, 2019.

[22] A. Norouzi, A. Emami, K. Najarian, N. Karimi, and S. R. Soroushmehr, "Exploiting uncertainty of deep neural networks for improving segmentation accuracy in MRI images," in Proceedings of the ICASSP 2019-2019 IEEE International Conference on Acoustics, Speech and Signal Processing (ICASSP), pp. 2322-2326, Brighton, UK, May 2019.

[23] W. Bai, M. Sinclair, G. Tarroni et al., "Automated cardiovascular magnetic resonance image analysis with fully convolutional networks," Journal of Cardiovascular Magnetic Resonance, vol. 20, no. 1, p. 65, 2018.

[24] H. B. Winther, C. Hundt, B. Schmidt et al., "U-net: deep learning for generalized biventricular cardiac mass and function parameters," 2017, https://arxiv.org/abs/1706.04397.

[25] D. Nie, J. Lu, H. Zhang et al., "Multi-channel 3D deep feature learning for survival time prediction of brain tumor patients using multi-modal neuroimages," Scientific Reports, vol. 9, no. 1, p. 1103, 2019.

[26] Y. Zhao, X. Li, W. Zhang et al., "Modeling 4d fMRI data via spatio-temporal convolutional neural networks (ST-CNN)," in Proceedings of the International Conference on Medical Image Computing and Computer-Assisted Intervention, pp. 181-189, Granada, Spain, September 2018.

[27] G. Ma, L. He, C.-T. Lu, P. S. Yu, L. Shen, and A. B. Ragin, "Spatio-temporal tensor analysis for whole-brain fMRI classification," in Proceedings of the 2016 SIAM International Conference on Data Mining, pp. 819-827, Miami, FL, USA, May 2016.

[28] M. Afshin, I. B. Ayed, A. Islam, A. Goela, T. M. Peters, and S. Li, "Global assessment of cardiac function using image statistics in MRI," in Proceedings of the International Conference on Medical Image Computing and Computer-Assisted Intervention, pp. 535-543, Nice, France, October 2012.

[29] M. Afshin, I. B. Ayed, K. Punithakumar et al., "Regional assessment of cardiac left ventricular myocardial function via MRI statistical features," IEEE Transactions on Medical Imaging, vol. 33, no. 2, pp. 481-494, 2013.

[30] Z. Wang, M. B. Salah, B. Gu, A. Islam, A. Goela, and S. Li, "Direct estimation of cardiac biventricular volumes with an adapted Bayesian formulation," IEEE Transactions on BioMedical Engineering, vol. 61, no. 4, pp. 1251-1260, 2014.

[31] X. Zhen, Z. Wang, A. Islam, M. Bhaduri, I. Chan, and S. Li, "Direct estimation of cardiac bi-ventricular volumes with regression forests," in Proceedings of the International Conference on Medical Image Computing and Computer-Assisted Intervention, pp. 586-593, Boston, MA, USA, September 2014.

[32] X. Zhen, Z. Wang, A. Islam, M. Bhaduri, I. Chan, and S. Li, "Multi-scale deep networks and regression forests for direct bi-ventricular volume estimation," Medical Image Analysis, vol. 30, pp. 120-129, 2016.

[33] X. Zhen, A. Islam, M. Bhaduri, I. Chan, and S. Li, "Direct and simultaneous four-chamber volume estimation by multi- output regression," in Proceedings of the International Conference on Medical Image Computing and Computer-Assisted Intervention, pp. 669-676, Munich, Germany, October 2015.

[34] A. Paszke, S. Gross, F. Massa et al., "Pytorch: an imperative style, high-performance deep learning library," in Proceedings of the Advances in Neural Information Processing Systems, pp. 8024-8035, Vancouver, Canada, December 2019. 